\title{
5-羟甲基糠醛加氢制备 1-羟基-2,5-己二酮的研究进展
}

\author{
许占威颜佩芳张宗超* \\ (中国科学院大连化学物理研究所 清洁能源国家实验室 催化基础国家重点实验室 大连 116023)
}

\begin{abstract}
摘要 生物质基平台化合物 5-羟甲基糠酫的转化研究是近年来的研究热点之一, 1-羟基-2,5-已二酮是一种具有广泛应 用前景的化合物. 由 5-羟甲基糠酫加氢制备 1-羟基-2,5-已二酮是一种重要的合成策略，具有绿色、原子经济性高等优 势, 取得了一些重要的研究进展. 从非均相催化和均相催化两方面对 1 -差基-2,5-已二酮的制备进行了综述, 讨论了催 化体系的优劣和催化机理的研究.
\end{abstract}

关键词 生物质; 呋喃; 氢化作用; 酮

\section{Advance in the Hydrogenation of 5-Hydroxymethylfurfural to Produce 1-Hydroxyhexane-2,5-dione}

\author{
$\mathrm{Xu}$, Zhanwei $\quad$ Yan, Peifang $\quad$ Zhang, Zongconrad* \\ (Dalian National Laboratory for Clean Energy, State Key Laboratory of Catalysis, Dalian Institute of Chemical Physics, \\ Chinese Academy of Sciences, Dalian 116023)
}

\begin{abstract}
Recently, catalytic transformation of biobased 5-hydroxymethylfurfural (5-HMF) has been paid much more attentions. 1-Hydroxyhexane-2,5-dione (HHD) is a potential feedstock to synthesize valuable chemicals. The recent remarkable progress of HHD preparation, which utilized 5-HMF as a starting material, is a green and atom economic method. In this review, the approaches of HHD preparation from 5-HMF are summarized based on the homogeneous and heterogeneous catalysis.
\end{abstract}

Keywords biomass; furan; hydrogenation; ketones

5-羟甲基糠醛 (5-HMF) 是近年来备受关注的化学品 之一, 可由来源于木质纤维素 ${ }^{[1,2]}$ 的果糖 ${ }^{[3]}$ 或葡萄糖 ${ }^{[4]}$ 经 脱水或异构化/脱水反应制得. 以 5-HMF 为平台化合物, 经氧化、还原、缩合等反应可制备多种高附加值的化工 产品(图 1) ${ }^{[5 \sim 13]}$. 5-HMF 的加氢产物 2,5-二羟甲基呋喃 (DHMF) 和 2,5-二羟甲基四氢呋喃(DHMHF)可作为聚合 物单体、5-HMF 的氧化产物 2,5-呋喃二甲酸(FDCA)被 认为是对苯二甲酸的替代物之一, 2,5-呋喃二甲醛(FDA) 可用来制备聚亚胺类材料, 2,5-二甲基呋喃(DMF)可作 为燃油添加剂, 5 - HMF 的还原胺化产物二[(5-差年甲基呋 喃基-2-)甲基]胺(BHMFA)可作为聚合物单体, 5-HMF 水 解产物乙酰丙酸(LA) 是一种应用前景广泛的平台化合
物，可以用来合成聚合物、燃料添加剂、药物分子等. 由 此可见, 开发基于 5-HMF 为原料的新型化学品对资源 化利用生物质显得尤为重要.

近年来, 有国内外研究组陆续报道了 $5-\mathrm{HMF}$ 加氢 水解制备 1-羟基-2,5-己二酮(HHD)的反应. HHD 是一种 2,5-已二酮类化合物, HHD 可能成为一种新型平台化合 物, 合成高附加值的化学品. 5-HMF 加氢水解制 HHD采 用了甲酸或氢气作为还原剂，均相催化剂如 $\mathrm{Ir} 、 \mathrm{Ru}$ 等配 合物表现出了高催化活性; 非均相催化剂如 $\mathrm{Pd} 、 \mathrm{Au}$ 等 也可高效催化该反应; 本文分别从非均相催化和均相催 化两个体系综述 HHD 合成方法，并对 HHD 潜在应用作 出展望.

\footnotetext{
* Corresponding author. E-mail: zczhang@yahoo.com
}

Received July 18, 2016; revised August 22, 2016; published online September 6, 2016

Project supported by the China Postdoctoral Science Foundation (No. 2013M540236), the Chinese Academy of Sciences/State Administration of Foreign Experts Affairs International Partnership Program for Creative Research Teams, and the Chinese Government "Thousand Talent” Program Funding. 中国博士后面上资助一等资助(No. 2013M540236)、中国科学院/国家外国专家局“创新团队国际合作伙伴计划”、国家千人计划资助项目. 


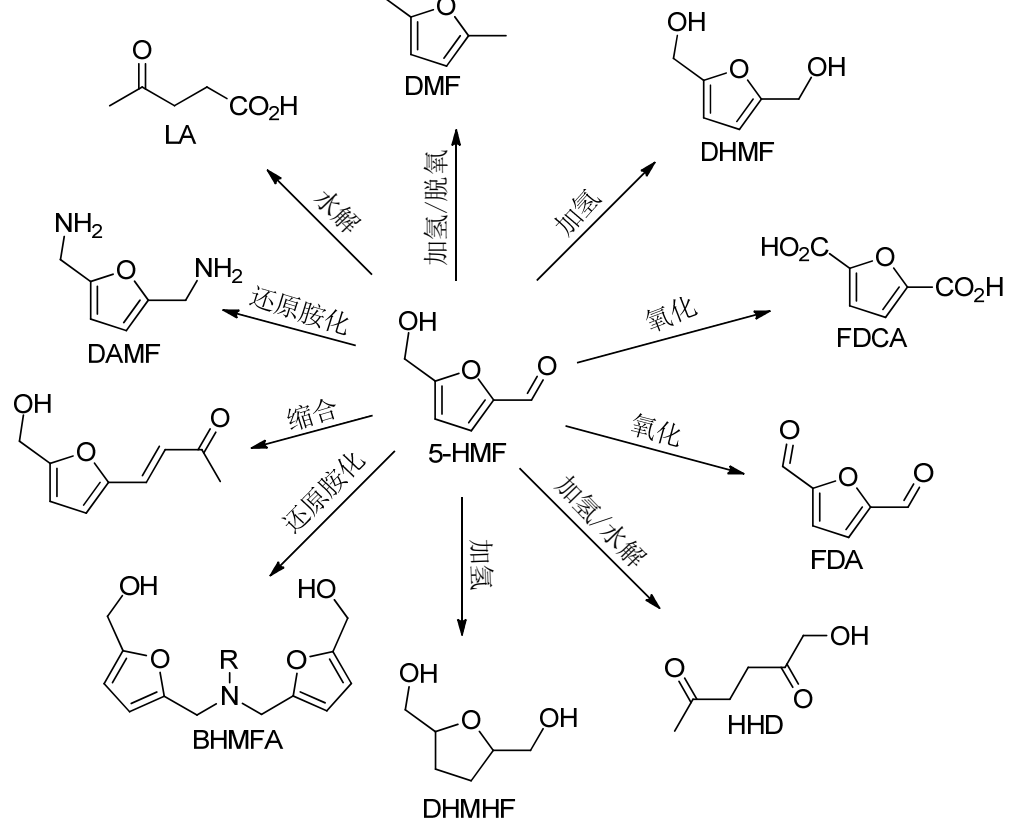

图 1 生物质基平台化合物 5-HMF 的转化

Figure 1 Chemical transformation of bio based 5-HMF

\section{HHD 的应用前景}

HHD 有两个酮羰基和一个伯醇羟基, 具有 2,5-己二 酮的反应性质 ${ }^{[14,15]}$, 能发生多种有机反应(图 2). 如与胺

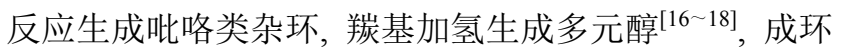
反应生成五元碳(氧杂)环或六元氧杂环 ${ }^{[19,20]}$. 虽然 HHD 的转化研究较少报道, 基于 HHD 的应用前景和制备 HHD 技术的进步, 未来 HHD 的转化研究受到越来越多 的关注.

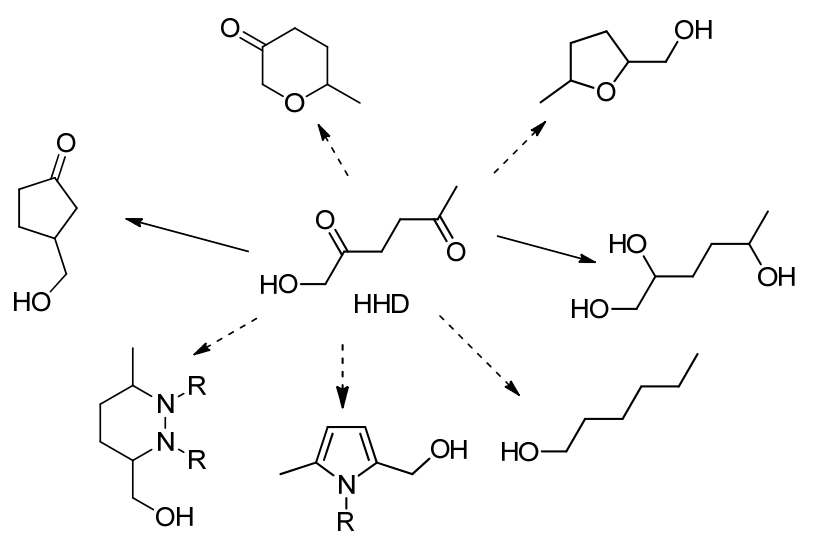

图 $2 \mathrm{HHD}$ 的应用前景

Figure 2 Chemical transformation of HHD

\section{2 非均相催化}

1991 年, Mentech 课题组 ${ }^{[21]}$ 研究了 $\mathrm{Pt} / \mathrm{C}$ 催化剂在草 酸存在下, 氢气为还原剂, 反应温度为 $140{ }^{\circ} \mathrm{C}$, HHD 收 率最高为 $60 \%$. 这是由 $5-\mathrm{HMF}$ 加氢制 HHD 的第一例报
道. 然而，随后的二十多年时间里，该反应并没有得到 足够重视. 其中一个可能的原因是生物质制备 5-HMF 一直是个难题. 直到由葡萄糖 ${ }^{[4]}$ 、果糖 ${ }^{[3]}$ 等六碳糖, 以及 纤维素 ${ }^{[22,23]}$ 合成 5-HMF 的突破, 才使得基于 5-HMF 的 化学转化包括由 5-HMF 加氢制 HHD 的研究逐渐被重视 起来.

Heeres 课题组 ${ }^{[24]}$ 报道了在 $\mathrm{Rh}-\mathrm{Re} / \mathrm{SiO}_{2}$ 催化剂的作 用下，HHD 的收率达到了 $81 \%$. 但是，该反应氢气压力 高(8.0 MPa), 反应时间长 $(17 \mathrm{~h})$. 徐杰课题组 ${ }^{[18]}$ 采用了 $\mathrm{Pd} / \mathrm{C}$ 作为催化剂, $\mathrm{H}_{3} \mathrm{PO}_{4}$ 作为酸催化剂, 在 $101 \mathrm{kPa}$ 的氢 气作用下, 水相中回流反应了 $72 \mathrm{~h}, \mathrm{HHD}$ 的收率为 $37 \%$. 低压氢气可能是 HHD 产率低的主要原因之一.

Jérôme 课题组 ${ }^{[25]}$ 报道了在 $\mathrm{Pd} / \mathrm{C}$ 催化剂作用下, 氢 气为还原剂, 水相中 5-HMF 制备 HHD, HHD 收率最高 为 $77 \%(\mathrm{Eq} .1)$. 该反应体系引入了高压的 $\mathrm{CO}_{2}$, 促进了 呋喃开环反应.

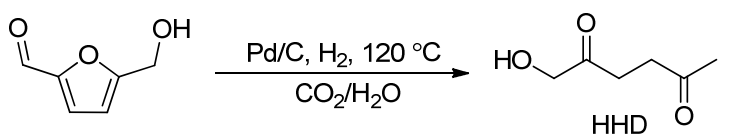

Jérôme 课题组提出了如 Scheme 1 的反应机理: $5-\mathrm{HMF}$ 在 $\mathrm{Pd} / \mathrm{C}$ 催化剂作用下加氢生成 DHMF, DHMF 与水反应生成中间体 $\mathbf{1}, \mathbf{1}$ 发生质子和羟基脱除生成中间 体 2, 2 在质子作用下发生呋喃开环反应生成 3.3 双键加 氢并烯醇式重排生成产物 HHD.

该反应过程中, 可检测到中间产物 DHMF; 以 DHMF 为原料, 在同样条件下, 可获得 $60 \%$ 的 HHD, 因 

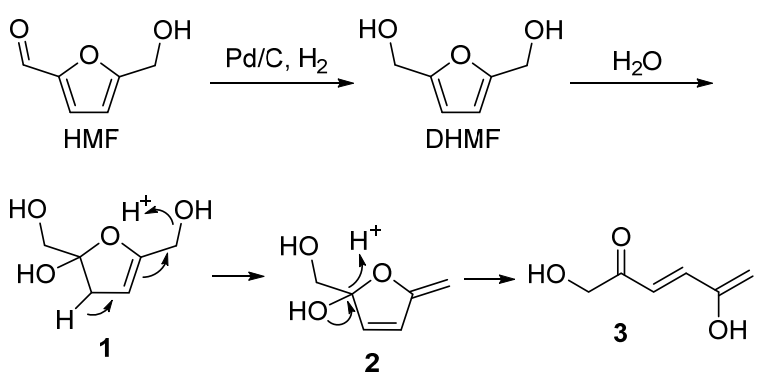<smiles>CC(=O)CCC(=O)CO</smiles>

图式 $1 \mathrm{Pd} / \mathrm{C}$ 催化的反应机理

Scheme 1 Reaction mechanism of $\mathrm{Pd} / \mathrm{C}$ catalysis

此证明了 DHMF 是反应的中间体(Scheme 2). 若没有还 原剂氢气, DHMF 开环生成的中间体 $\mathbf{4}$ 非常不稳定, 可 能与 DHMF 通过 Diels-Alder 反应生成结构复杂的聚合 物胡敏素, 这条反应途径是导致 HHD 选择性低的主要 因素之一。

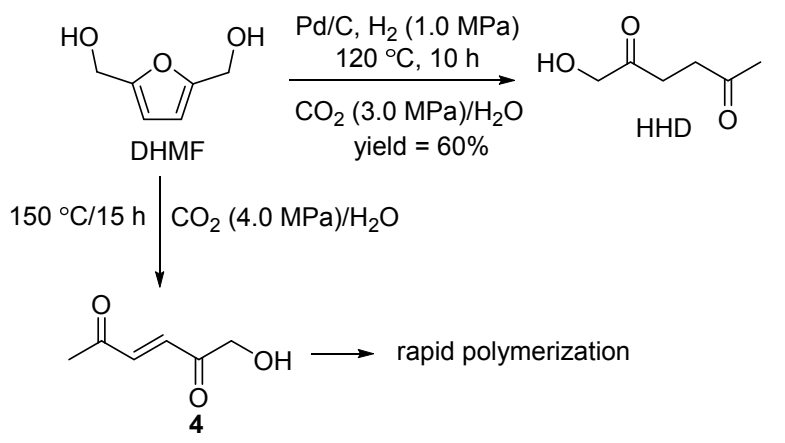

图式 2 DHMF 的反应活性研究

Scheme 2 Reactivity study of DHMF

$\mathrm{CO}_{2} / \mathrm{H}_{2} \mathrm{O}$ 作为一种温和的酸体系, 催化了羟基脱 除、呋喃开环等过程, 而果糖在酸催化作用下可脱水生 成 5-HMF. 以果糖或菊粉为原料, 通过一锅多步反应, 合成了 HHD，总产率分别为 $36 \%$ 或 $15 \%$.

Jérôme 课题组 ${ }^{[26]}$ 也报道了 Amberlyst-15 和 $\mathrm{Pd} / \mathrm{C}$ 在 THF 中可高效催化 5-HMF 加氢制备 HHD, 反应收率最 高为 $77 \%$. 该体系反应温度仅为 $80{ }^{\circ} \mathrm{C}$, 但需要较高的 氢气压力 $(5.0 \mathrm{MPa})$. 该体系也同样实现了以果糖或菊粉 为原料制备 $\mathrm{HHD} . \mathrm{Pd} / \mathrm{C}$ 体系虽然催化效率高, 但由于 HHD 形成中常伴随着胡敏素的形成, 胡敏素容易聚集 在固体催化剂上, 可能导致催化剂失活. 因此如何再生 $\mathrm{Pd} / \mathrm{C}$ 催化剂可能是该体系存在的关键问题.

Ohyama 课题组 ${ }^{[27]}$ 以 $\mathrm{HAuCl}_{4}$ 为催化剂前体, 采用沉 淀法合成了不同金属氧化物载体负载的非均相 $\mathrm{Au}$ 催化 剂, 并研究了水中非均相 $\mathrm{Au}$ 催化剂催化的 5-HMF 加氢 反应, 可以选择性获得 HHD 或者环戊酮衍生物(Scheme
3). 酸性氧化物载体负载的 $\mathrm{Au}$ 催化剂催化效果好于碱 性氧化物负载的 $\mathrm{Au}$ 催化剂, 说明酸性氧化物载体有利 于中间产物 DHMF 的开环反应. 其中氧化物载体五氧 化二铌 $\left(\mathrm{Nb}_{2} \mathrm{O}_{5}\right)$ 展现出了最好的催化效果，3-羟甲基环戊 酮收率可达 $86 \%$. 催化剂反应结束后，经简单的清洗干 燥处理, 仍保持了高活性. $\mathrm{Nb}_{2} \mathrm{O}_{5}$ 的作用是催化了呋喃 环开环以及 HHD 的分子内 Aldol 缩合生成环戊酮的反 应. 在 $\mathrm{Au} / \mathrm{Nb}_{2} \mathrm{O}_{5}$ 催化体系中加入 $\mathrm{H}_{3} \mathrm{PO}_{4}$, 降低溶液 $\mathrm{pH}$ 可抑制 Aldol 缩合反应, 使加氢产物停留在 HHD, 收率 可达 $60 \%$. 在确定了最佳的 $\mathrm{Nb}_{2} \mathrm{O}_{5}$ 作为催化剂载体后, 也研究了负载其它类型金属 $\mathrm{Ru} 、 \mathrm{Pd}$ 和 Pt 的催化效果, 结 果表明 $\mathrm{Au}$ 的催化效果最高.

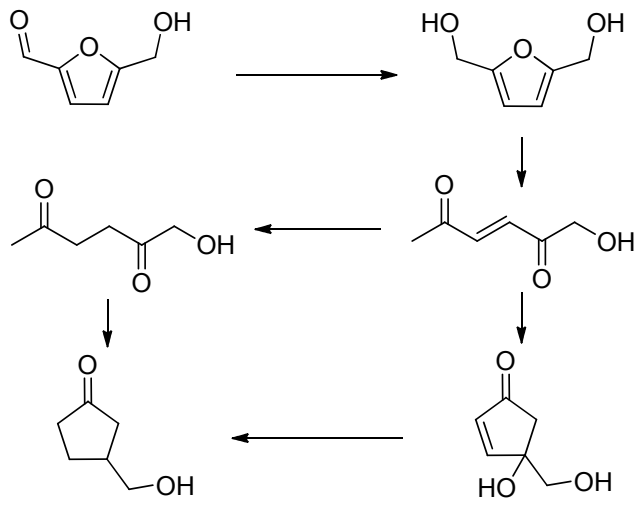

图式 $3 \mathrm{Au}$ 催化 5-HMF 加氢环合反应

Scheme 3 Hydrogenation-cyclization of 5-HMF catalyzed by Au catalyst

目前, 非均相催化体系的研究报道仅有以上几例, 而且采用甲酸作为氢源的研究未见报道. 开发廉价金属 负载的非均相催化剂, 充分考虑载体在水中稳定性, 实 现 5-HMF 加氢制 HHD, 以及非均相催化剂的循环使用 对 HHD 产业化至关重要.

\section{3 均相催化}

我们课题组 ${ }^{[28]}$ 报道了 $\mathrm{Cp} *$ Ir 配合物催化的 5 -HMF 加氢水解制 HHD 的反应(Scheme 4), 合成了一系列吡啶 型配体配位的 $\mathrm{Cp} * \mathrm{Ir}$ 配合物. Ir 催化剂用量为 5-HMF 的 $0.26 \mathrm{~mol} \%$, HHD 收率最高为 $78 \%$. 联吡啶配体 5 配位的 Cp*Ir 配合物催化活性最高, HHD 收率为 $78 \%$. 提高氢 气压力或温度都可以提高 HHD 产率. Cp*Ir 配合物作为 加氢催化剂催化了加氢过程, 同时与氢气反应形成的质 子催化了水解过程.

该工作研究了反应中间产物的活性，表明 DHMF 为 HHD 形成的中间体. 另外, 通过同位素示踪实验和 基于统计学的计算提出了 $\mathrm{Cp} * \mathrm{Ir}$ 催化的反应机理 (Scheme 5): 首先 5-HMF 的醛基被加氢生成 DHMF, 


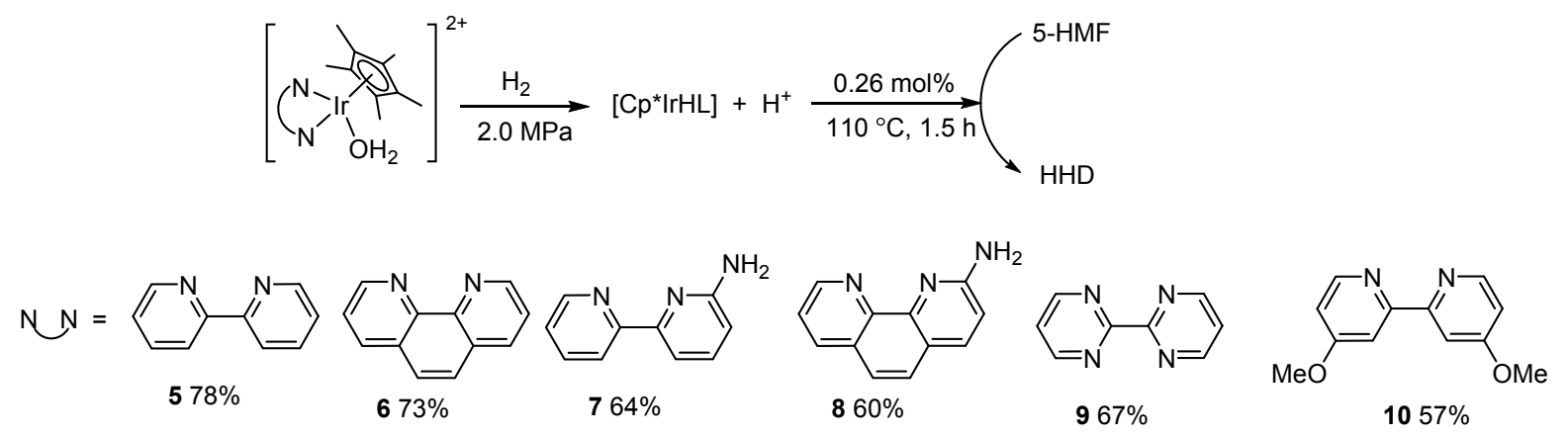

图式 $4 \mathrm{Cp} *$ Ir 催化 5-HMF 水相中加氢

Scheme $4 \mathrm{Cp}$ *Ir-catalyzed hydrogenation of 5-HMF in aqueous solution

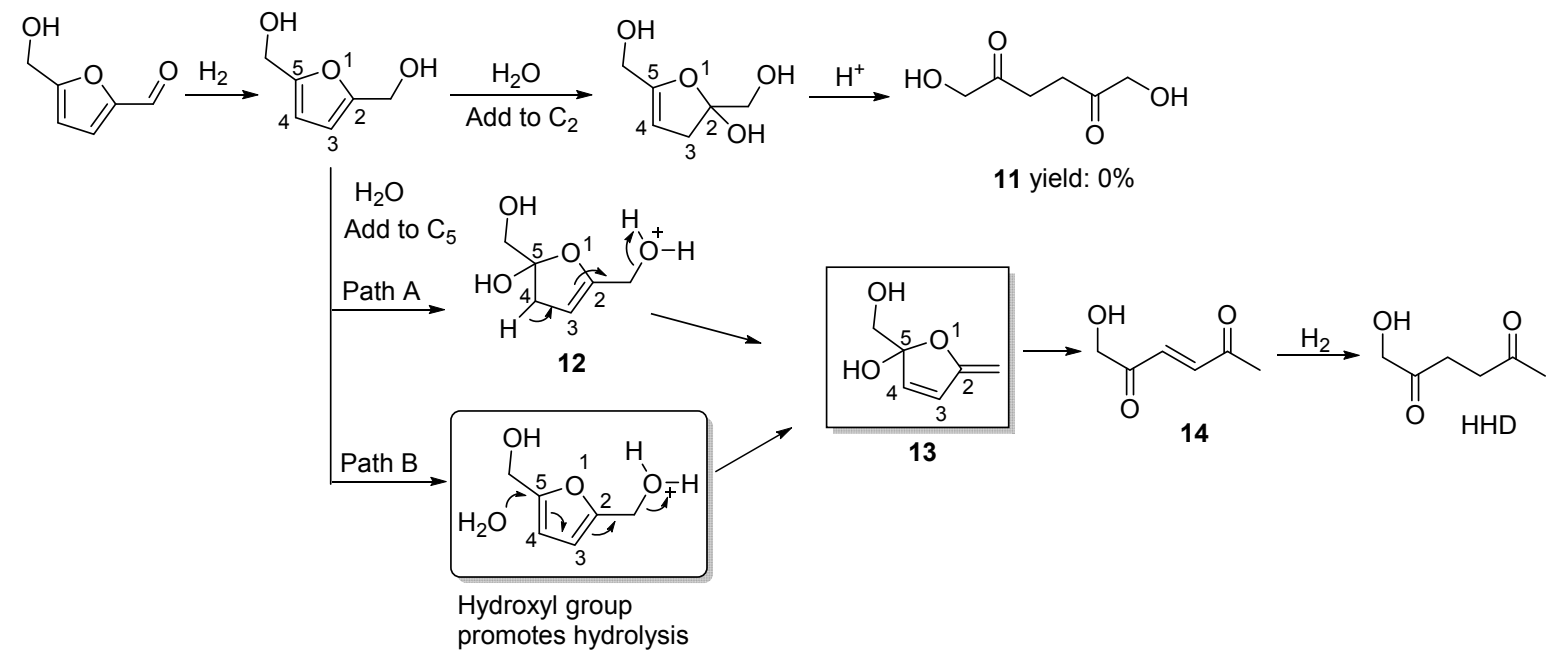

图式 $5 \mathrm{Cp}$ *Ir 催化 5-HMF 加氢制 HHD 的反应机理

Scheme 5 Mechanism of Cp*Ir-catalyzed hydrogenation of 5-HMF

DHMF 发生羟基促进的水解反应脱除了羟基生成 13 (Path B), DHMF 也可先水解再脱除质子和羟基生成 $\mathbf{1 3}$ (Path A), 中间体 13 发生开环加氢生成产物 HHD. Path $\mathrm{A}$ 在反应中经历了中间体 12, 12 引入了溶剂中的质子, 因此, 研究了在 $\mathrm{D}_{2} \mathrm{O}$ 中 DHMF 的反应活性. 通过 GC-MS 研究产物 HHD 中的氛分布, 采用统计计算的方 法模拟了两种途径 HHD 产物的氛分布(表 1). Path B 路 径模拟的结果接近实验结果, 因此, HHD 的形成经历了 Path B.

$\mathrm{Cp}$ *Ir 配合物催化活性高, 条件相对于非均相催化 更为温和, 然而 Ir 昂贵的价格可能会限制其在大规模制 备 HHD 中的应用. 一种解决的办法是制备活性更高的 $\mathrm{Cp}$ *Ir 加氢配合物, 提高催化剂的转化数 (TON) 和转化 频率(TOF).

差基官能化的联吡啶配体在碱性条件下表现出了 极高的催化加氢活性 ${ }^{[29-36]}$. 羟基的作用是: (1)邻位羟基 协助金属 Ir 活化氢气, (2)羟基在碱性条件下脱质子形成 的氧负离子给电子能力强, (3)羟基的水溶性有利于水相
中的催化反应. 5-HMF 加氢制 HHD 的反应过程包含了 酸催化过程, 增加溶液 $\mathrm{pH}$ 会降低 HHD 选择性. 而酸性 条件下 $\mathrm{NMe}_{2}$ 给电子能力要强于 $\mathrm{OH}$. 因此, 我们 ${ }^{[37]}$ 设计 了新的联吡啶配体 19, 结合了羟基的活化氢气作用和 $\mathrm{NMe}_{2}$ 的强给电子作用(图 3), 同时，也考察其它取代基 $\left(\mathrm{CF}_{3} 、 \mathrm{Me}\right)$ 对 $\mathrm{Cp}$ * Ir 催化加氢活性的影响. 结果表明, 给 电子基增加了 $\mathrm{Cp}$ * Ir 催化加氢活性, 而吸电子基降低了 Cp*Ir 催化加氢活性; 19 配位的 Cp*Ir-19 催化活性最高, HHD 的 TON 值达到 70800, TOF 值达到 $31560 \mathrm{~h}^{-1}$, 均 是目前文献报道的最高值. Cp*Ir-19 也适用于甲酸作为 氢源的反应, HHD 收率为 $61.4 \%$, TOF 值达到 $6140 \mathrm{~h}^{-1}$. 可见，配体 19 对于催化加氢活性的提升起到了决定作 用(Scheme 6): $\mathrm{Cp} * \mathrm{Ir}-19$ 的羟基脱除质子形成氧负离子 中间体 20, 中间体 $\mathbf{2 0}$ 形成酮式结构 21. 中间体 $\mathbf{2 0}$ 与氢 气反应经历了水参与的环状过渡态实现了氢气的异裂 形成中间体 22, 22 通过 8 元环过渡态 23 与醛基或双键 反应生成产物和中间体 21, 并进入下一个催化循环. 
表 1 产物気分布的实验与计算数据

Table 1 Deuterium distribution

\begin{tabular}{|c|c|c|c|c|}
\hline & DHMF & $\mathrm{HHD}$ & $15(m / z=99)$ & \\
\hline Data resource & $\mathrm{D}_{1}-\mathbf{1 5}(\mathrm{m} / \mathrm{z}=100)$ & $\mathrm{D}_{2}-\mathbf{1 5}(m / z=101)$ & $\mathrm{D}_{3}-\mathbf{1 5}(m / z=102)$ & $\mathrm{D}_{4}-\mathbf{1 5}(m / z=103)$ \\
\hline GC-MS & $5.8 \%$ & $70.1 \%$ & $20.8 \%$ & $2.7 \%$ \\
\hline Calculation (Path A) & $0.2 \%<\omega<2.5 \%$ & $6.9 \%<\omega<38.9 \%$ & $46.8 \%<\omega<69.4 \%$ & $11.7 \%<\omega<23.5 \%$ \\
\hline Calculation (Path B) & $4.7 \%$ & $70.7 \%$ & $24.5 \%$ & $0 \%$ \\
\hline
\end{tabular}

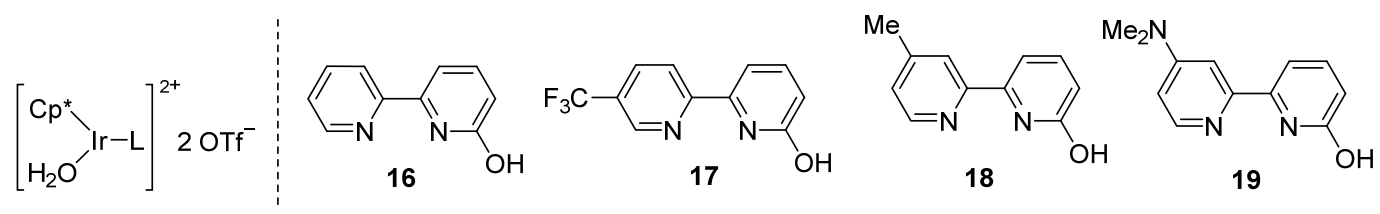

图 $3 \mathrm{Cp}$ * Ir 配合物的结构

Figure 3 Structure of $\mathrm{Cp}$ * Ir complexes

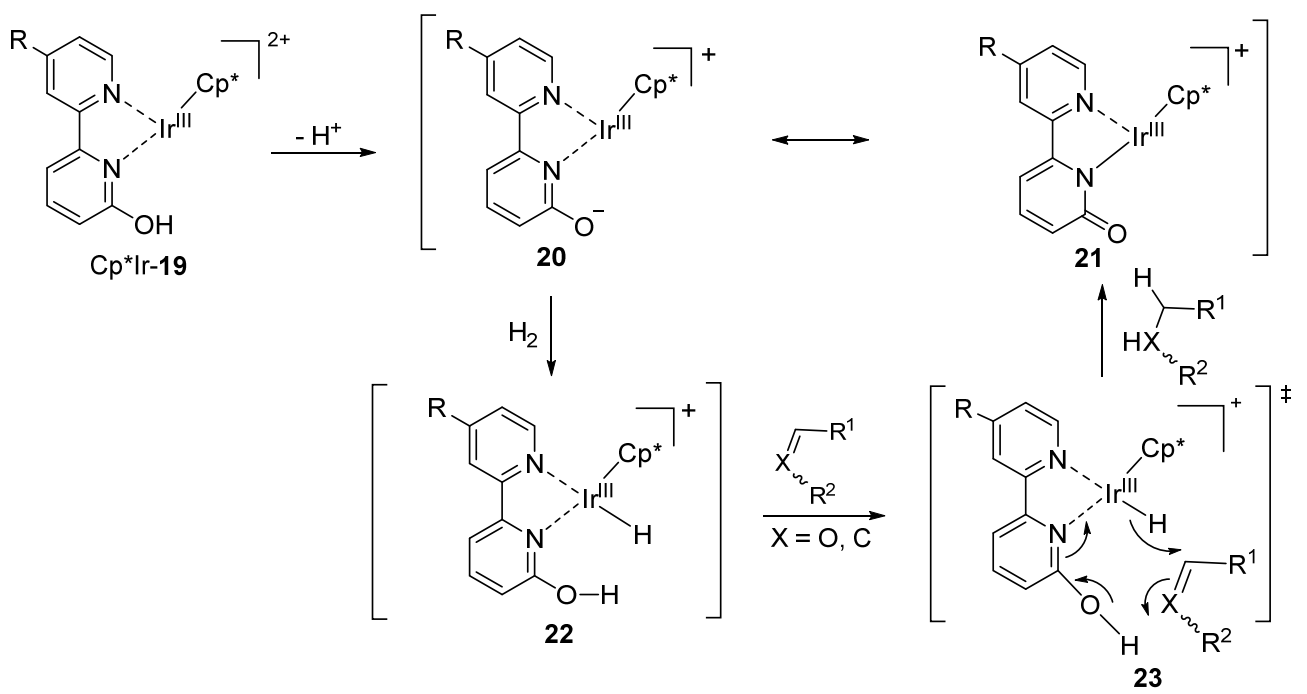

图式 $6 \mathrm{Cp}$ *Ir 催化加氢机理

Scheme 6 Mechanism of Cp*Ir-catalyzed hydrogenation

甲酸可来源于生物质, 是纤维素生产乙酰丙酸(LA) 的产物之一, 且储存和运输方便, 因此甲酸作为绿色氢 源的加氢反应也受到了关注. 傅尧课题组 ${ }^{[38]}$ 首先报道 了 $\mathrm{Cp}$ * Ir 配合物为催化剂, 甲酸为氢源, 5-HMF 加氢制 HHD. 当联吡啶上取代基为 $\mathrm{OH}$ 或 $\mathrm{OMe}_{2}$ 时, 催化活性 高(图 4). 溶剂 $\mathrm{pH}$ 决定了加氢选择性: 当 $\mathrm{pH}$ 小于 4 时, HHD 为主要加氢产物; 当 $\mathrm{pH}$ 大于 4 时, DHMF 为主要 加氢产物; $\mathrm{pH}$ 为 2.5 时, $\mathrm{HHD}$ 的产物高达 99\%. 而采用 氢气作为还原剂时, HHD 的收率为 $70 \%$ 左右 $\left(4 \mathrm{MPa} \mathrm{H}_{2}\right)$. 他们也研究了催化剂的循环使用和 HHD 的分离: 采用 二氯甲烷作为萃取剂, 在反应结束后可实现 HHD 和水
相催化剂的分离：水相催化剂可继续下一次循环，但 $\mathrm{Cp}^{*} \mathrm{Ir}$ 催化剂会有少部分失活导致 HHD 的产率有所下 降, $\mathrm{Cp}$ * Ir 催化剂失活的原因是中间体对氧气不稳定所 致.

傅尧课题组认为，在甲酸体系中，呋喃环内氧原子 协助了醇羟基的离去(Scheme 7), 形成的中间体也有利 于呋喃的开环反应.

除了 Ir 配合物展示了很高的催化活性外, Ru 配合物 也可以催化 5-HMF 加氢制 HHD，但活性仍不及 Ir 配合 物. Singh 课题组 ${ }^{[39]}$ 报道了水相中, 甲酸为氢源, Ru 配合 物催化 5-HMF 形成 HHD(图 5). 甲酸用量为 5-HMF 的 


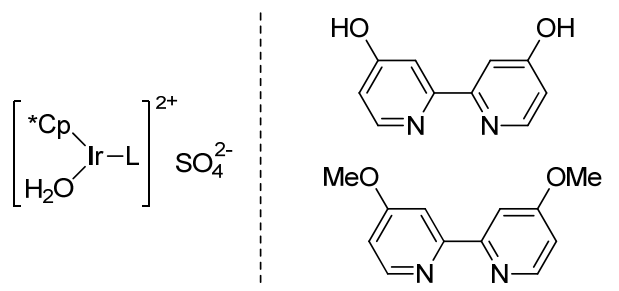

图 4 甲酸体系中 $\mathrm{Cp}$ * Ir 催化剂结构

Figure 4 Structure of $\mathrm{Cp}$ * Ir complexes in formic acid system

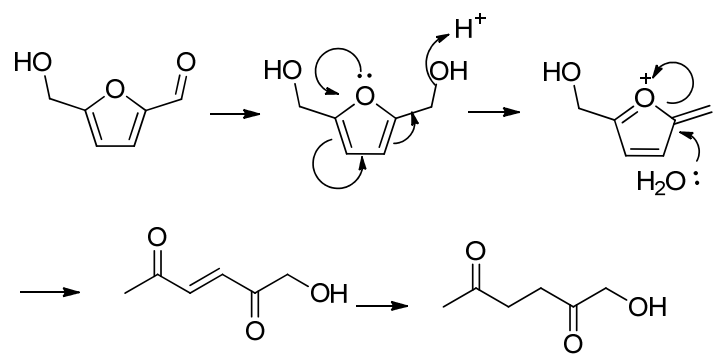

图式 7 甲酸体系中 $\mathrm{Cp}$ * Ir 催化剂结构

Scheme 7 Structure of $\mathrm{Cp}^{*} \mathrm{Ir}$ complexes in formic acid system

12 倍量, 温度为 $80{ }^{\circ} \mathrm{C}$, 在催化剂 25 作用下, 5-HMF 完 全转化, HHD 的选择性达到 $52 \%$.

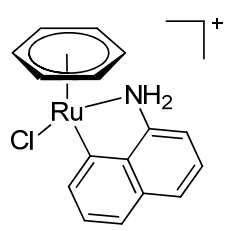

24

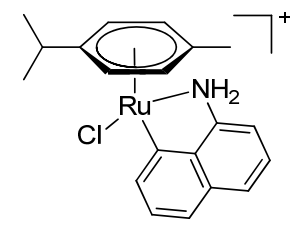

25
图 $5 \mathrm{Ru}$ 催化剂结构

Figure 5 Structure of $\mathrm{Ru}$ complexes

目前, 均相催化剂的研究仅限于 Ir、Ru 两类. Ir 催 化活性和选择性均好于 $\mathrm{Ru}$ 催化剂, 但是价格昂贵的 $\mathrm{Ir}$ 催化剂可能会限制其应用, 开发合适的分离方法仍然是 Ir 催化的方向之一. Ru 均相催化的研究还处于起步阶 段, 开发新的配体, 研究氢气为氢源的反应活性, 将是 $\mathrm{Ru}$ 催化今后的研究方向.

\section{4 结论与展望}

综上所述, HHD 是一种应用前景广阔的化合物. 以 生物质基平台化合物 5-HMF 为原料合成 HHD 的路线环 境友好, 经济效益高. 非均相催化体系产物、催化剂分 离容易, 但是催化效率低, 催化剂的失活, 再生还没有 较为系统的研究. 均相催化体系研究中, Ir 配合物的活 性高、选择性高, 但是产物分离、催化剂循环使用较为 困难. $\mathrm{Ru}$ 配合物的催化活性低, 研究也仅处于起步阶段. 开发均相催化体系的有效分离方法, 提高 $\mathrm{Ru}$ 配合物的 催化活性, 将是今后可能的发展方向之一. 另外, 研究 $\mathrm{HHD}$ 的应用, 开发基于 HHD 的精细化学品, 也将是未
来研究的重点之一.

\section{References}

[1] Corma, A.; Iborra, S.; Velty, A. Chem. Rev. 2007, 107, 2411.

[2] Lynd, L. R.; Wyman, C. E.; Gerngross, T. U. Biotechnol. Prog. 1999, 15, 777.

[3] Román-Leshkov, Y.; Chheda, J. N.; Dumesic, J. A. Science 2006, 312, 1933.

[4] Zhao, H.; Holladay, J. E.; Brown, H.; Zhang, Z. C. Science 2007, $316,1597$.

[5] Teong, S. P.; Yi, G.; Zhang, Y. Green Chem. 2014, 16, 2015.

[6] Wang, J.; Zhang, C.-P.; Ouyang, P.-K. Chem. Ind. Eng. Prog. 2008, 27,702 (in Chinese).

(王军, 张春鹏, 欧阳平凯, 化工进展, 2008, 27, 702.)

[7] Sheldon, R. A. Green Chem. 2014, 16, 950.

[8] van Putten, R.-J.; van der Waal, J. C.; de Jong, E.; Rasrendra, C. B.; Heeres, H. J.; de Vries, J. G. Chem. Rev. 2013, 113, 1499.

[9] Zakrzewska, M. E.; Bogel-Łukasik, E.; Bogel-Łukasik, R. Chem. Rev. 2011, 111, 397.

[10] Bozell, J. J.; Petersen, G. R. Green Chem. 2010, 12, 539.

[11] Nakagawa, Y.; Tamura, M.; Tomishige, K. ACS Catal. 2013, 3, 2655.

[12] Zhu, S.; Wang, J.; Fan, W. Catal. Sci. Technol. 2015, 5, 3845.

[13] Xu, Z.; Yan, P.; Liu, K.; Wan, L.; Xu, W.; Li, H.; Liu, X.; Zhang, Z. C. ChemSusChem 2016, 9, 1255.

[14] O'Reilly, E.; Iglesias, C.; Ghislieri, D.; Hopwood, J.; Galman, J. L.; Lloyd, R. C.; Turner, N. J. Angew. Chem., Int. Ed. 2014, 53, 2447.

[15] Kotani, S.; Aoki, S.; Sugiura, M.; Ogasawara, M.; Nakajima, M. Org. Lett. 2014, 16, 4802.

[16] Sharma, G. V. M.; Srikanth, G.; Reddy, P. P. Org. Biomol. Chem. 2012, 10, 8119.

[17] Chen, J.; Lu, F.; Zhang, J.; Yu, W.; Wang, F.; Gao, J.; Xu, J. ChemCatChem 2013, 5, 2822.

[18] Yang, Y.; Du, Z.; Ma, J.; Lu, F.; Zhang, J.; Xu, J. ChemSusChem 2014, 7, 1352 .

[19] Shono, T.; Matsumura, Y.; Hamaguchi, H. J. Chem. Soc., Chem. Commun. 1977, 712.

[20] Chatterjee, M.; Ishizaka, T.; Kawanami, H. Green Chem. 2014, 16 , 1543.

[21] Schiavo, V.; Descotes, G.; Mentech, J. Bull. Soc. Chim. Fr. 1991, 704.

[22] Rout, P. K.; Nannaware, A. D.; Prakash, O.; Kalra, A.; Rajasekharan, R. Chem. Eng. Sci. 2016, 142, 318.

[23] Putro, J. N.; Soetaredjo, F. E.; Lin, S.-Y.; Ju, Y.-H.; Ismadji, S. RSC Adv. 2016, 6, 46834.

[24] Buntara, T.; Noel, S.; Phua, P. H.; Meliá-Cabrera, I.; de Vries, J. G.; Heeres, H. J. Angew. Chem., Int. Ed. 2011, 50, 7083.

[25] Liu, F.; Audemar, M.; De Oliveira Vigier, K.; Clacens, J.-M.; De Campo, F.; Jérôme, F. ChemSusChem 2014, 7, 2089.

[26] Liu, F.; Audemar, M.; De Oliveira Vigier, K.; Clacens, J.-M.; De Campo, F.; Jérôme, F. Green Chem. 2014, 16, 4110.

[27] Ohyama, J.; Kanao, R.; Esaki, A.; Satsuma, A. Chem. Commun. 2014, 50, 5633.

[28] Xu, Z.; Yan, P.; Xu, W.; Liu, X.; Xia, Z.; Chung, B.; Jia, S.; Zhang, Z. C. ACS Catal. 2015, 5, 788.

[29] Fujita, K.; Tanaka, Y.; Kobayashi, M.; Yamaguchi, R. J. Am. Chem. Soc. 2014, 136, 4829.

[30] Zeng, G.; Sakaki, S.; Fujita, K.; Sano, H.; Yamaguchi, R. ACS Catal. 2014, 4, 1010 .

[31] Fujita, K.; Kawahara, R.; Aikawa, T.; Yamaguchi, R. Angew. Chem., Int. Ed. 2015, 54, 9057. 
[32] Wang, W.-H.; Himeda, Y.; Muckerman, J. T.; Manbeck, G. F.; Fujita, E. Chem. Rev. 2015, 115, 12936.

[33] Wang, W.-H.; Ertem, M. Z.; Xu, S.; Onishi, N.; Manaka, Y.; Suna, Y.; Kambayashi, H.; Muckerman, J. T.; Fujita, E.; Himeda, Y. ACS Catal. 2015, 5, 5496.

[34] Onishi, N.; Xu, S.; Manaka, Y.; Suna, Y.; Wang, W.-H.; Muckerman, J. T.; Fujita, E.; Himeda, Y. Inorg. Chem. 2015, 54, 5114.

[35] Wang, R.; Ma, J.; Li, F. J. Org. Chem. 2015, 80, 10769.
[36] Ou, W. C.; Cundari, T. R. ACS Catal. 2015, 5, 225.

[37] Xu, Z.; Yan, P.; Li, H.; Liu, K.; Liu, X.; Jia, S.; Zhang, Z. C. ACS Catal. 2016, 6, 3784

[38] Wu, W.-P.; Xu, Y.-J.; Zhu, R.; Cui, M.-S.; Li, X.-L.; Deng, J.; Fu, Y. ChemSusChem 2016, 9, 1209.

[39] Gupta, K.; Tyagi, D.; Dwivedi, A. D.; Mobin, S. M.; Singh, S. K. Green Chem. 2015, 17, 4618. 